EPJ Web of Conferences 41, 08014 (2013)

DOI: $10.1051 /$ epjconf/20134108014

C) Owned by the authors, published by EDP Sciences, 2013

\title{
Nonclassical energy transfer in photosynthetic FMO complex
}

\author{
Vytautas Abramavicius $^{1}$ and Darius Abramavicius ${ }^{1,2, a}$ \\ 1 Department of Theoretical Physics, Vilnius University, Sauletekio al. 9-III, 10222 Vilnius, Lithuania \\ 2 State Key Laboratory of Supramolecular Structure and Materials, Jilin University, Changchun 130012, \\ China.
}

\begin{abstract}
Excitation energy transfer in a photosynthetic FMO complex has been simulated using the stochastic Schrödinger equation. Fluctuating chromophore transition energies are simulated from the quantum correlation function which allows to properly include the finite temperature. The resulting excitation dynamics shows fast thermalization of chromophore occupations into proper thermal equilibrium. The relaxation process is characterized by entropy dynamics, which shows nonclassical behavior.
\end{abstract}

\section{Introduction}

Energy transfer in photosynthetic aggregates drives the biological energy cycle on earth initiated by the photosynthesis process. The photosynthetic molecular aggregates participating in such processes are essentially the open quantum systems that strongly interact with the heterogeneous protein environment [1]. This interaction leads to pronounced dissipation effects thus causing irreversible energy dynamics and transport [2].

The energy transport in photosynthetic aggregates has received a lot of attention due to nontrivial interplay between the protein fluctuations and quantum relaxation of chromophore excitations [3]. The transport phenomena are usually described using approximate methods of the reduced density matrix $[2,4]$. However, these theories may miss specific important effects such as quantum transport. Additionally they are dependent on the chosen basis set. The exact methods such as hierarchical equations of motion or the path-integral techniques avoid these problems but require a lot of computational power[6,5]. Additionally, the density matrix describes the ensemble averaged system information. However the explicit dynamics of the individual FMO system affected by fluctuating environment contains important information about the quality of energy transfer in a single molecular aggregate. The stochastic methods are necessary to account for such explicit dynamics[7]. In this paper we study the energy transport in the photosynthetic Fenna-Matthews-Olson (FMO) aggregate [8] using the stochastic Schrödinger equation. Characteristics of the system are obtained by calculating the chromophore populations and the system entropy. We also compare the results calculated using the Debye (or the over damped Brownian oscillator) spectral density [9] and the one obtained from FMO molecular dynamics simulations [10].

\section{Model}

We consider a system described by the Frenkel Exciton Hamiltonian. For our present simulations only single excitation is sufficient to include thus the Hamiltonian of the isolated system can be written as

\footnotetext{
a e-mail: darius.abramavicius@ff.vu.1t
}

This is an Open Access article distributed under the terms of the Creative Commons Attribution License 2.0, which permits unrestricted use, distribution, and reproduction in any medium, provided the original work is properly cited. 
[9]

$$
\hat{H}_{S}=\sum_{i}^{N} E_{i}^{(S)}|i\rangle\left\langle i\left|+\sum_{i \neq j}^{N} J_{i j}^{(S)}\right| i\right\rangle\langle j| .
$$

Here $i$ label different chromophores, $E_{i}^{(S)}$ are chromophore transition energies and $J_{i j}^{(S)}$ are interchromophore dipole-dipole interactions. The environment (heat bath) is described by the infinite number of uncoupled harmonic oscillators, which are linearly coupled to the system and induce the fluctuations of the chromophore energies. These fluctuations are completely characterized by the correlation function [9]. For the energy of the $i$-th chromophore we have the correlation function

$$
C_{i}(t)=\int \frac{d \omega}{2 \pi} C_{i}^{\prime \prime}(\omega)(\operatorname{coth}(\beta \omega / 2) \cos (\omega t)-i \sin (\omega t))
$$

Here $\beta=\hbar /\left(k_{\mathrm{B}} T\right)$. The spectral density function $C_{i}^{\prime \prime}(\omega)$ here describes the frequency distribution of the bath.

When the system and the bath are uncorrelated at some initial time the system wavefunction $\left|\psi_{Z}(t)\right\rangle$ satisfies the stochastic Schrödinger equation [12]

$$
\frac{\mathrm{d}}{\mathrm{d} t}\left|\psi_{Z}(t)\right\rangle=-i \widehat{H}_{S}\left|\psi_{Z}(t)\right\rangle+\sum_{i}|i\rangle\left\langle i\left|\left(Z_{i}(t)-\int_{0}^{t} d s C_{i}(t-s) \frac{\delta}{\delta Z_{i}(t)}\right)\right| \psi_{Z}(t)\right\rangle .
$$

Here $Z_{i}(t)$ is a Gaussian stochastic trajectory with correlation function $\left\langle Z_{i}^{*}(t) Z_{i}(s)\right\rangle_{Z}=C_{i}(t-s)$ of Eq. 2. Additional properties correspond to the properties of the quantum harmonic bath: $\left\langle Z_{i}(t)\right\rangle_{Z}=0$ and $\left\langle Z_{i}(t) Z_{i}(s)\right\rangle_{Z}=0$. The trajectory $Z(t)$ thus mimics the action of the stochastic force.

An arbitrary stochastic trajectory can be simulated from the known correlation function with the help of the Wiener-Khinchin theorem giving [11]

$$
Z_{i}(t)=\int \frac{d \omega}{2 \pi} \exp \left(i \omega t+\phi_{i}(\omega)\right) \sqrt{C_{i}^{\prime \prime}(\omega)(1+\operatorname{coth}(\beta \omega / 2))}
$$

where $\phi_{i}(\omega)$ is a stochastic zero-correlated function of frequency. Given realizations of $\phi_{i}(\omega)$, the state vectors $\left|\psi_{Z}(t)\right\rangle$ can be numerically propagated using Eq. 3 .

The ensemble-averaged observables can then be recovered as statistical mean values. For instance the reduced density matrix $\widehat{\rho}_{S}=\left\langle\mid \psi_{Z}(t)\right\rangle\left\langle\psi_{Z}(t) \mid\right\rangle_{Z}$. Its diagonal values $\left.\widehat{\rho}_{S}\right)_{i i}$ are state populations. Their time evolution reflects non equilibrium system dynamics.

\section{Results}

We apply the stochastic Schrödinger equation to calculate the excitation energy transfer dynamics in the FMO monomer of seven units at room temperature [9]. Protein dynamics of FMO has been studied by Olbrich et al [10] and the average spectral density has been evaluated (we denote this type of spectral density as the Olbrich spectral density. Here we compare the exciton dynamics affected by the bath of either Olbrith [10] or of the Debye [9] spectral densities. The memory-integral in Eq. 3 has been neglected for simplicity.

Excitation populations in the FMO monomer in the chromophore representation is shown in Fig. 1. Surprisingly we find that the evolutions of both spectral densities are similar, while weak oscillations of the populations emerge in the case of the Debye spectral density. We do not observe population oscillations with the Olbrich spectral density due to interference with its resonances. In both cases the equilibrium is reached approximately after $1 \mathrm{ps}$. The final distribution of populations in the chromophore basis is close to Boltzmanian which shows that our model relaxes to the proper thermal equilibrium.

To characterize the non-equilibrium behavior of the system we additionally calculate the quantum entropy function defined by

$$
S(t)=-\operatorname{Tr}\left(\widehat{\rho}_{r e d} \ln \widehat{\rho}_{r e d}\right)
$$



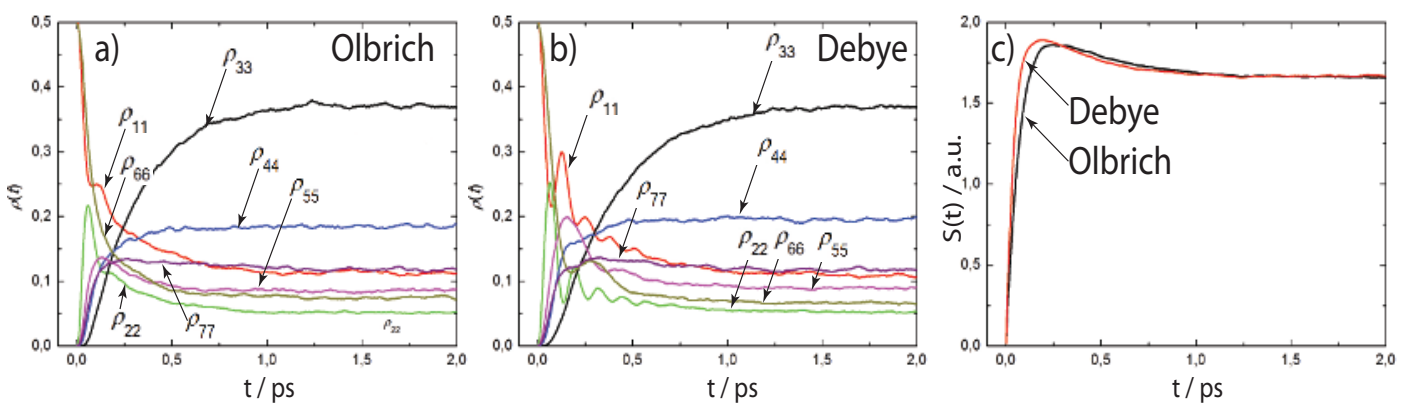

Fig. 1. a) FMO populations calculated with Olbrich spectral density, b) debye spectral density. c) FMO exciton entropies.

(the Boltzmann constant is omitted here). As is known from classical non-equilibrium dynamics, the system evolves in the direction of increasing entropy until its maximum is reached. The entropies of the FMO using the two bath models are depicted in Fig. 1c. We find that the entropy of the system increases rapidly in the first $200 \mathrm{fs}$ and then it slowly decreases to its equilibrium value. The relaxation time is the same as the time needed for equilibration. This rise-and-decay behavior of the entropy suggests that quantum effects play a significant role in the system.

\section{Conclusions}

In conclusion we found that the population dynamics in the chromophore basis relaxes to the proper thermal equilibrium, thus the excitonic couplings hardly maintain exciton delocalization in the process of energy transport. The dynamics of the excitation in the FMO monomer calculated using the realistic Olbrich spectral density do not differ qualitatively from the results obtained with Debye spectral density, thus, the usage of the simpler spectral density is justified. Last, the non-classical behavior (initial steep rise and later decay) of the system entropy suggests the quantum effects contributing to the energy transfer.

\section{References}

1. H. van Amerogen, L. Valkunas, R. van Grondelle, Photosynthetic Excitons (World Scien- tific, Singapore, 2000).

2. V. May, O Kühn, Charge and Energy Transfer Dynamics in Molecular Systems, 2nd ed., (WileyVCH, Weinheim, 2004).

3. M. Mohseni, P. Rebentrost, S. Lloyd, A. Aspuru-Guzik, J. Chem. Phys. 129, (2008) 174106.

4. D. Abramavicius, V. Butkus, L. Valkunas, In: E. R. Weber, C. Jagadish, Series editors, Semiconductors and Semimetals, 85. Quantum Efficiency in Complex Systems, Part II: From Molecular Aggregates to Organic SolarCells, ed. U. Würfel, M. Thorwart, E. R.Weber. (San Diego: Academic Press, 2011) 3-46.

5. R. Hützen, S. Weiss, M. Thorwart, R. Egger, Phys. Rev. B 85, (2012) 121408.

6. J. Strümpfer, K. Schulten, J. Chem. Theory Comput. doi:dx.doi.org/10.1021/ct3003833.

7. H. P. Breuer, F. Petruccione, The Theory of Open Quantum Systems, (Oxford University Press, New York, 2007).

8. M. T. Milder, B. Brüggemann, R. van Grondelle, J. L. Herek. Photosynth Res. 104, (2010) 257.

9. D. Abramavicius, B. Palmieri, D. V. Voronine, F. Sanda, S. Mukamel, Chem. Rev. 109, (2009) 2350.

10. C. Olbrich, J. Strümpfer, K. Schulten, U. Kleinekathoefer, J. Phys. Chem. Lett. 2, (2011) 1771.

11. D. Abramavicius, L. Valkunas. Phys. Rev. B 68, (2003) 245203.

12. L. Diósi, W. T. Strunz, Phys. Lett. A 235, (1997) 569. 